\title{
Blockchain y mercados financieros: aspectos generales del impacto regulatorio de la aplicación de la tecnología blockchain en los mercados de crédito de América Latina Blockchain and Financial Markets: General Features of the Regulatory Impact of the Application of Blockchain Technology in the Credit Markets of Latin America
}

\author{
JORGE ARMANDO CORREDOR HIGUERA* \\ Universidad Externado de Colombia
}

\section{DAVID DÍAZ GUZMÁN** \\ Universidad Externado de Colombia}

Resumen: La finalidad de este documento es analizar, a partir de criterios tecnológicos, estándares regulatorios y recomendaciones internacionales, el impacto de la tecnología blockchain en los mercados de crédito y cómo dicha dinámica podría tener efectos directos en los mercados latinoamericanos, a saber, producir mayor eficiencia e inclusión financiera en la región. Para tal fin, se contextualizará el alcance de la tecnología blockchain, se desarrollarán las posibles aplicaciones de dicha tecnología en los mercados financieros y, por último, se analizarán las propuestas regulatorias sobre la aplicación de dicha tecnología en los mercados de crédito latinoamericanos en lo relacionado con la administración de riesgos e inclusión financiera.

Palabras clave: blockchain, mercados de créditos, inclusión financiera, riesgos financieros, eficiencia

Abstract: The purpose of this document is to analyze, based on technological criteria, regulatory standards and international recommendations, the impact of blockchain technology on credit markets and how such dynamics could have direct effects on Latin American markets, i.e., producing greater efficiency and financial inclusion in the region. For this purpose, the scope of blockchain technology will be contextualized, the possible applications of said technology in the financial markets will be developed, and finally

* Este documento es un artículo de investigación realizado como parte de la línea de investigación de intervención del Estado y autorregulación, dentro de los productos de investigación desarrollados por el Observatorio de Derecho Financiero y del Mercado de Valores de la Universidad Externado de Colombia.

** Abogado de la Universidad Nacional de Colombia, especialista en derecho privado económico de la Universidad Nacional de Colombia, LL.M. Derecho internacional, inversiones, comercio y arbitraje impartido por la Universidad de Heidelberg - Universidad de Chile. Docente investigador del Departamento de Derecho Financiero y Bursátil de la Universidad Externado de Colombia y profesor en pregrado y posgrado de la Facultad de Derecho de la Universidad Externado de Colombia. Código ORCID: 0000-0003-4672-1789. Correo electrónico: jorgearmando.corredor@gmail.com

*** Abogado de la Universidad Externado de Colombia, candidato a Magíster en Derecho Económico de la Universidad Externado de Colombia. Asistente de investigación del Departamento de Derecho Financiero y Bursátil de la Universidad Externado de Colombia y docente en pregrado del Departamento de Matemáticas de la misma universidad.

Código ORCID: 0000-0002-4167-0153. Correo electrónico: david.diaz@uexternado.edu.co 
the regulatory proposals on the application of said technology in the Latin American credit markets will be analyzed in relation to risk management and financial inclusion.

Key words: blockchain, credit markets, financial inclusion, financial risks, efficiency

CONTENIDO: I. INTRODUCCIÓN.- II.TECNOLOGÍA BLOCKCHAIN.- II.1. ALCANCE DE LA TECNOLOGÍA.- II.2. VENTAJAS DE LA TECNOLOGÍA BLOCKCHAIN EN RELACIÓN CON LOS SISTEMAS CENTRALIZADOS.- II.3. CRIPTOMONEDAS Y BLOCKCHAIN.- II.4. FORMAS DE LA TECNOLOGÍA BLOCKCHAIN.- II.5. CONSENSO EN LA CADENA DE BLOQUES.- II.6. CONTRATOS INTELIGENTES O SMART CONTRACTS.- III. APLICACIÓN DEL BLOCKCHAIN EN LOS MERCADOS FINANCIEROS.- III.1. MERCADO DE VALORES.- III.2. MERCADO DE SEGUROS.III.3. MERCADO DE CRÉDITO.- IV. PROPUESTAS REGULATORIAS PARA LA APLICACIÓN DE BLOCKCHAIN EN LOS MERCADOS DE CRÉDITO EN AMÉRICA LATINA Y SU IMPACTO EN EL MERCADO.- IV.1. VISIÓN DE UN ENFOQUE PARTICIPATIVO POR PARTE DE LOS REGULADORES FINANCIEROS Y LAS EMPRESAS QUE ESTRUCTURAN PROCESOSTECNOLÓGICOS DE BLOCKCHAIN.IV.2. REGULACIÓN EN MATERIA DE RIESGOS.- IV.3. PROTECCIÓN DE DATOS.IV.4. IMPACTO DE LA APLICACIÓN DE TECNOLOGÍA DE BLOCKCHAIN EN LOS MERCADOS DE CRÉDITO LATINOAMERICANOS.- V. CONCLUSIONES.

\section{INTRODUCCIÓN}

El presente trabajo pretende establecer los posibles impactos de la aplicación de la tecnología blockchain (cadena de bloques) en los mercados de crédito latinoamericanos y cómo estos podrían verse beneficiados por dicha tecnología en temas relacionados a la liquidez, eficiencia y mayor acceso a sus productos por parte de la población con bajos niveles de acceso a servicios financieros. Ahora bien, la tecnología de la cadena de bloques surgió como un mecanismo por medio del cual se busca el intercambio de información y la estructuración de procesos a través de mecanismos descentralizados con nodos de información interconectados, justificados en esquemas criptográficos. Una de las ventajas de la tecnología blockchain es que permite que los procesos sean más ágiles, seguros, transparentes y que se realicen con una significativa reducción de costos en relación con procesos centralizados de información. La tecnología blockchain representa hoy en día una revolución en el mundo entero por el posible impacto que puede traer en sectores tales como administración de justicia, salud y educación, servicios notariales, procesos de supervisión por parte de órganos de control, auditorías, ejecución de contratos, monedas virtuales, mercados financieros, funcionamiento de empresas y de entidades estatales, entre otros. 
La tecnología de la cadena de bloques se empezó a estructurar como soporte de las criptomonedas, en especial del Bitcoin, pero tanto el sector privado como los Estados han encontrado un potencial en dicha tecnología, sin que los mercados financieros sean la excepción. De este modo, dentro de los mercados de capital, la tecnología blockchain puede tener aplicaciones tanto en la prestación de bienes y servicios que ofrecen las instituciones financieras como en el desarrollo de los procesos internos de las entidades y en las actividades de inspección, vigilancia y control del supervisor financiero. Por otra parte, una posible respuesta al problema de los bajos niveles de acceso a los servicios financieros en América Latina podría ser la aplicación de la tecnología blockchain con el fin de dar acceso a una gran parte de la población a los servicios financieros. Por tal razón, a partir de procesos de regulación, se puede potencializar la aplicación de la tecnología blockchain con el fin de generar eficiencia, entrada al mercado y acceso a los recursos financieros de los que un alto porcentaje de la población latinoamericana carece actualmente.

Bajo los anteriores presupuestos, el presente trabajo busca desarrollar la siguiente pregunta de investigación: ¿Cuáles podrían ser las propuestas regulatorias para América Latina frente a la aplicación de la tecnología blockchain en los mercados de crédito?

Sobre la base de esta pregunta se establece una hipótesis según la cual, a partir de un enfoque participativo entre las autoridades regulatorias, instituciones financieras y empresas de tecnología financiera (industria conocida como Fintech), se busca desarrollar aplicaciones de tecnología blockchain al mercado de crédito, bajo estándares de mitigación de riesgos operativos, legales, informáticos y de privacidad, con lo cual, se dinamizará la liquidez de los mercados y el acceso a los servicios financieros. Como objetivo general de la investigación, se busca establecer propuestas regulatorias para la aplicación de la tecnología blockchain en los mercados de crédito en América Latina. Los objetivos específicos de este documento son (i) identificar las diversas aplicaciones de la tecnología blockchain que existen para los mercados de crédito; (ii) proponer estándares para la mitigación de riesgos operativos, legales, informáticos y de privacidad en el uso de la tecnología blockchain para los mercados de crédito; (iii) demostrar que la aplicación de la tecnología blockchain en los mercados de crédito dinamiza la liquidez del mercado y facilita el acceso a los servicios financieros.

La metodología de investigación de este trabajo consistió en el análisis de fuentes bibliográficas e información pública. El trabajo es de tipo descriptivo y de enfoque cualitativo, pues tiene el propósito de explicar las características del tema tratado con fundamento en el análisis de las fuentes de información. Por la complejidad de la política pública a

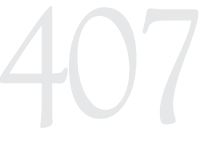

BLOCKCHAIN Y MERCADOS FINANCIEROS: ASPECTOS

GENERALES

DEL IMPACTO

REGULATORIO DE

LA APLICACIÓN DE

LA TECNOLOGÍA

BLOCKCHAIN EN

LOS MERCADOS

DE CRÉDITO DE

AMÉRICA LATINA

BLOCKCHAIN

AND FINANCIAL

MARKETS: GENERAL

FEATURES OF

THE REGULATORY

IMPACT OFTHE

APPLICATION

OF BLOCKCHAIN

TECHNOLOGY INTHE CREDIT MARKETS OF LATIN AMERICA 
desarrollar y por las limitaciones de este documento, se propondrá de forma general algunas posibles soluciones adicionales de intervención estatal.

El documento presentará las generalidades de la tecnología blockchain haciendo alusión a los diferentes campos de aplicación de esta. En segundo lugar, se presentarán las principales aplicaciones que a la fecha se han materializado de la tecnología blockchain en los mercados de crédito. En tercer lugar, se presentarán algunas propuestas regulatorias sobre la aplicación de dicha tecnología en los mercados de crédito latinoamericanos con relación a la administración de riesgos e inclusión financiera. Por último, se enunciarán algunas conclusiones.

\section{TECNOLOGÍA BLOCKCHAIN II.1. Alcance de la tecnología}

Las aplicaciones que podría tener el blockchain se encuentran en proceso de desarrollo debido a los potenciales que representa dicha tecnología (Government Office for Science, 2016). Así, tanto el sector público como el privado están incursionando en la aplicación de dicha tecnología, en escenarios tales como contratación, administración de justicia, registros públicos, procesos de flujo de productos y servicios a través de cadenas de suministros, entre otros (Natarajan, Krause \& Gradstei, 2017, p. IX). Con respecto a la definición de lo que se entiende por la tecnología blockchain, se tomarán algunos conceptos establecidos por expertos en la materia en la actualidad, sin desconocer que dicho concepto sigue en proceso de constitución. Tapscott y Tapscott determinan lo siguiente:

¿Qué pasaría si existiera un internet del valor en el que las partes de una transacción pudieran almacenar e intercambiar valor sin la necesidad de intermediarios tradicionales? En pocas palabras, eso es lo que ofrece la tecnología blockchain. El valor no se guarda en un archivo en algún lugar; se representa mediante transacciones registradas en una hoja de cálculo global o libro mayor, que aprovecha los recursos de una gran red peer-to-peer para verificar y aprobar transacciones. Un blockchain tiene varias ventajas. Primero, es distribuido: se ejecuta en computadoras proporcionadas por voluntarios de todo el mundo, por lo que no existe una base de datos central para piratear. En segundo lugar, es público: cualquiera puede verlo en cualquier momento porque reside en la red. Y tercero, está encriptado: usa encriptación de alto rendimiento para mantener la seguridad (2017, pp. 10-11).

En igual sentido, Swan establece lo siguiente:

Blockchain es un libro mayor descentralizado y transparente con los registros de transacciones — una base de datos que es compartida por 
todos los nodos de la red, actualizada por los mineros, monitoreada por todos, y de propiedad y controlada por nadie. Es como una hoja de cálculo interactiva gigante a la que todos tienen acceso y actualizaciones, y confirma que las transacciones digitales que transfieren fondos son únicas $(2015$, p. 1).

BLOCKCHAIN

Y MERCADOS

FINANCIEROS:

ASPECTOS

GENERALES

contable distribuido, descentralizado, público y encriptado, en el cual las personas pueden almacenar información y hacer transacciones seguras sin la necesidad de intermediarios. La información de las transacciones no está guardada en un archivo central, está representada por transacciones registradas en una hoja de cálculo global o libro mayor que aprovecha los recursos de una gran red peer-to-peer para verificar y aprobar transacciones (2015).

\section{Gráfico 1}

\section{LEDGER}

\section{Clave encriptado}

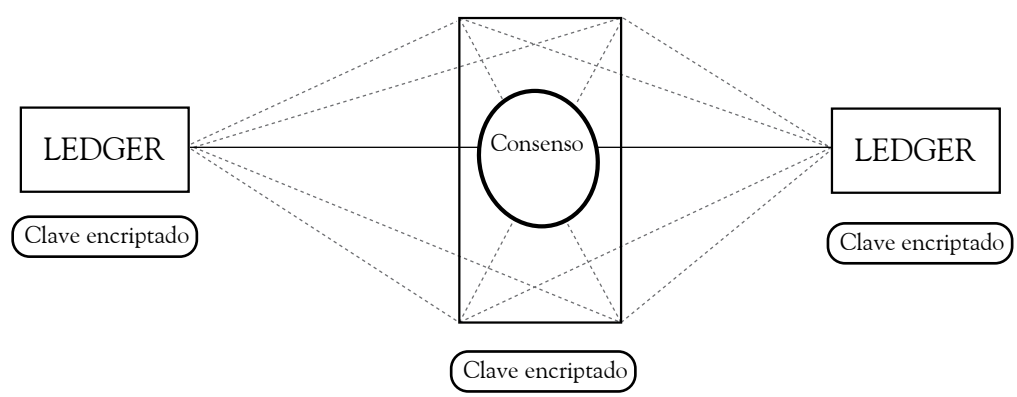

LEDGER
DEL IMPACTO

REGULATORIO DE

LA APLICACIÓN DE

LATECNOLOGÍA

BLOCKCHAIN EN

LOS MERCADOS

DE CRÉDITO DE

AMÉRICA LATINA

BLOCKCHAIN

AND FINANCIAL

MARKETS: GENERAL

FEATURES OF

THE REGULATORY

IMPACT OFTHE

APPLICATION

OF BLOCKCHAIN

TECHNOLOGY INTHE

CREDIT MARKETS OF

LATIN AMERICA

\section{II.2. Ventajas de la tecnología blockchain en relación con los sistemas centralizados}

A continuación, se procederá a establecer las principales características de los sistemas centralizados y descentralizados con el fin de determinar las ventajas del sistema descentralizado. 


\section{Tabla 1}

\begin{tabular}{|c|c|c|}
\hline Característica & Sistema centralizado & Blockchain \\
\hline $\begin{array}{l}\text { Administración } \\
\text { de la información }\end{array}$ & $\begin{array}{l}\text { Existe un administrador de } \\
\text { la información. }\end{array}$ & $\begin{array}{l}\text { La información se en- } \\
\text { cuentra descentralizada. }\end{array}$ \\
\hline $\begin{array}{l}\text { Sistema de segu- } \\
\text { ridad }\end{array}$ & $\begin{array}{l}\text { El administrador debe im- } \\
\text { plementar un sistema de se- } \\
\text { guridad con la finalidad de } \\
\text { proteger la información. } \\
\text { La estructuración de los me- } \\
\text { canismos de seguridad está } \\
\text { en manos del administrador } \\
\text { o de un tercero señalado } \\
\text { por este, sin que, por ello, el } \\
\text { administrador deje de ser el } \\
\text { responsable. }\end{array}$ & $\begin{array}{l}\text { Existe un sistema cripto- } \\
\text { gráfico, el cual puede va- } \\
\text { riar a través de los meca- } \\
\text { nismos de claves públicas } \\
\text { y privadas. }\end{array}$ \\
\hline Transparencia & $\begin{array}{l}\text { El administrador establece } \\
\text { los mecanismos por medio } \\
\text { de los cuales los participan- } \\
\text { tes acceden a la totalidad } \\
\text { de la información dentro de } \\
\text { protocolos establecidos para } \\
\text { tal fin. }\end{array}$ & $\begin{array}{l}\text { Los participantes del sis- } \\
\text { tema tienen la posibilidad } \\
\text { de acceder a la informa- } \\
\text { ción y verificarla a través } \\
\text { de la cadena de bloques. }\end{array}$ \\
\hline Costos & $\begin{array}{l}\text { Se materializan costos por } \\
\text { razón de la infraestructura } \\
\text { tecnológica y en materia de } \\
\text { la ciberseguridad que requie- } \\
\text { re el administrador central } \\
\text { en el manejo de la informa- } \\
\text { ción. }\end{array}$ & $\begin{array}{l}\text { Hay una reducción de } \\
\text { costos, ya que el manejo } \\
\text { de la información es re- } \\
\text { emplazado por códigos } \\
\text { algorítmicos, los cuales, a } \\
\text { través de nodos, procesan } \\
\text { y verifican la información } \\
\text { de forma independiente } \\
\text { de cada transacción. }\end{array}$ \\
\hline $\begin{array}{l}\text { Alterabilidad de la } \\
\text { información }\end{array}$ & $\begin{array}{l}\text { Depende de los sistemas tec- } \\
\text { nológicos de ciberseguridad } \\
\text { con que cuenta el admi- } \\
\text { nistrador, los cuales no son } \\
\text { inmunes a ataques ciberné- } \\
\text { ticos. }\end{array}$ & $\begin{array}{l}\text { Al existir una descentrali- } \\
\text { zación de la información, } \\
\text { la cual está organizada en } \\
\text { bloques por medio de pro- } \\
\text { cesos algorítmicos, la ma- } \\
\text { nipulación y alteración } \\
\text { de dicha información es } \\
\text { difícil de realizar. }\end{array}$ \\
\hline
\end{tabular}

Fuente: Elaborada sobre la base de Preukschat (2017). 


\section{II.3. Criptomonedas y blockchain}

Se ha entendido que las criptomonedas (criptodivisas o criptoactivos) son una representación digital de un valor. Estas pueden ser comercializadas digitalmente y tienen las características de unidad de cuenta, unidad de intercambio y unidad de uso, pero no cuentan con la característica de uso legal forzoso en ningún Estado (The Financial Action Task Force, 2015, p. 26).

Dentro de las principales características de las criptomonedas se pueden destacar las siguientes: «a) no tienen representación física; b) son descentralizadas, esto es, no están bajo el control de ningún Estado o entidad financiera; c) tienen carácter internacional; d) son anónimas, permitiendo preservar la privacidad en las transacciones; e) no necesitan intermediarios; f) tienen una función aceleradora ya que otorgan agilidad a los intercambios y a las operaciones de pago» (Pacheco Jiménez, 2016, p. 7). Ahora bien, en la actualidad no existe claridad sobre cuántas criptomonedas hay en el mercado, debido a la creación periódica de dichos activos ${ }^{1}$. No obstante, se describirán de forma general las principales criptomonedas que existen en el mercado, sin entrar a analizar a fondo cada una de ellas, ya un análisis de este tipo escaparía la finalidad de esta investigación.

\section{Tabla 2}

\begin{tabular}{|l|l|}
\hline \multicolumn{1}{|c|}{ Criptomonedas } & \multicolumn{1}{c|}{ Descripción } \\
\hline Bitcoin & $\begin{array}{l}\text { Criptomoneda más transada. } \\
\text { Utiliza sistema público. } \\
\text { Plataforma descentralizada. } \\
\text { Verificación se realiza a través de mineros. }\end{array}$ \\
\hline Etherum & $\begin{array}{l}\text { Ejecuta contratos inteligentes. } \\
\text { Permite crear mercados, nuevos productos y servicios a } \\
\text { través de los contratos inteligentes. }\end{array}$ \\
\hline Bitcoin Cash & $\begin{array}{l}\text { Dinero electrónico. } \\
\text { Sistema efectivo electrónico peer to peer. }\end{array}$ \\
\hline Ripple & $\begin{array}{l}\text { Mecanismo para enviar dinero globalmente a través de } \\
\text { blockchain. } \\
\text { Utilizado por las instituciones para procesar los pagos de } \\
\text { sus clientes en cualquier parte del mundo en tiempo real } \\
\text { y confiable. }\end{array}$ \\
\hline
\end{tabular}

BLOCKCHAIN

Y MERCADOS

FINANCIEROS:

ASPECTOS

GENERALES

DEL IMPACTO

REGULATORIO DE

LA APLICACIÓN DE

LATECNOLOGÍA

BLOCKCHAIN EN

LOS MERCADOS

DE CRÉDITO DE

AMÉRICA LATINA

\section{BLOCKCHAIN}

AND FINANCIAL

MARKETS: GENERAL

FEATURES OF

THE REGULATORY

IMPACT OF THE

APPLICATION

OF BLOCKCHAIN

TECHNOLOGY INTHE CREDIT MARKETS OF LATIN AMERICA

1 A partir de la información encontrada en internet para la fecha de elaboración de este documento, se tenía como dato que existían alrededor de 1400 criptodivisas en el mercado (http://financialtranslator.com/tag/cuantas-criptomonedas-hay/). 
Ahora bien, además de las diferentes criptomonedas que existen en la actualidad, hoy en día nos encontramos ante el hecho de la financiación de grandes proyectos empresariales a través de la infraestructura tecnológica blockchain ICO token (Pastor Sempere, 2017). Frente a este punto, se entra en una discusión con respecto a la naturaleza jurídica de los token. De esta manera, se debate si dichos token tienen la naturaleza de valores, en los términos de la regulación del mercado de valores. Así, en el caso de los Estados Unidos, la Comisión de Bolsa y Valores (U.S. Securities and Exchange Commission (SEC)) determinó que los ICO pueden ser ofertados en los términos de la regulación del mercado de valores, motivo por el cual se debe contar con la autorización de dicha entidad y deben regirse por las reglas de registro y negociación de los valores (SEC, 2018).

En este escenario, es necesario realizar algunas aclaraciones sobre los siguientes conceptos.

- Cadena de bloques: se entiende como un registro de contabilidad pública, por medio del cual se muestran las transacciones confirmadas. Permite que el usuario puede tener un número determinado de criptomonedas. Es importante señalar en este punto que tanto la integridad como el orden cronológico de la cadena de bloques se materializa a través de la criptografía (Pacheco Jiménez, 2016, p. 6).

- Minería: por medio de la minería «se transmiten y confirman las transacciones pendientes a ser incluidas en la cadena de bloques. Este proceso hace cumplir un orden cronológico en la mencionada cadena, protege la neutralidad de la red y permite un acuerdo entre todos los equipos sobre el estado del sistema. Para confirmar las transacciones deberán ser unidas en un bloque que se ajuste a estrictas normas de cifrado y que será verificado por la red, lo que impedirá que cualquier bloque anterior se modifique» (Pacheco Jiménez, 2016, p. 6).

- Token: es un protocolo descentralizado que toma forma como una cadena alfanumérica y representa un registro en la base de datos descentralizada de consenso de Bitcoin (Pastor Sempere, 2017, p. 299). En palabras de Preukschat, el token es una serie de dígitos que representa un registro de las cadenas de bloques, siendo estrictamente un sistema descentralizado (2017). 


\section{II.4. Formas de la tecnología blockchain}

El World Economic Forum determina que, a la fecha, existen tres tipos de Distributed-Ledger Technology², a saber: i) el sistema público, ii) el sistema privado y iii) un sistema híbrido (World Economic Forum, 2018, p. 5). La siguiente tabla presenta las principales características de estos sistemas:

\section{Tabla 3}

\begin{tabular}{|c|c|c|c|}
\hline Característica & Sistema público & Sistema privado & $\begin{array}{l}\text { Sistema } \\
\text { híbrido }\end{array}$ \\
\hline Definición & $\begin{array}{l}\text { En este sistema cual- } \\
\text { quier parte está en la } \\
\text { posibilidad de parti- } \\
\text { cipar en el proceso } \\
\text { sin ningún tipo de } \\
\text { verificación (Deloit- } \\
\text { te, } 2017 \mathrm{~b}, \text { p. } 4 \text { ). } \\
\text { Cada unidad de } \\
\text { cuenta se denomina } \\
\text { token. }\end{array}$ & $\begin{array}{l}\text { Existe un adminis- } \\
\text { trador que evalúa } \\
\text { la participación de } \\
\text { un agente dentro } \\
\text { del sistema. }\end{array}$ & $\begin{array}{l}\text { La mezcla de } \\
\text { los sistemas } \\
\text { públicos y } \\
\text { privados: los } \\
\text { nodos son pri- } \\
\text { vados, pero las } \\
\text { transacciones } \\
\text { se hacen de } \\
\text { forma públi- } \\
\text { ca (Rochina, } \\
2017 \text { ). }\end{array}$ \\
\hline Riesgos & $\begin{array}{l}\text { La verificación de } \\
\text { la operación está en } \\
\text { manos de los mine- } \\
\text { ros, sin que sea posi- } \\
\text { ble que otras partes } \\
\text { participen en el pro- } \\
\text { ceso de verificación. } \\
\text { No existen criterios } \\
\text { para verificar los } \\
\text { antecedentes de los } \\
\text { participantes del pro- } \\
\text { ceso. } \\
\text { Hay una mayor posi- } \\
\text { bilidad de que se ma- } \\
\text { terialicen problemas } \\
\text { de lavado de activos. } \\
\text { Problemas en mate- } \\
\text { ria de privacidad }\end{array}$ & $\begin{array}{l}\text { Mayor protección } \\
\text { de la información } \\
\text { de los participantes } \\
\text { Los usuarios están } \\
\text { sujetos a una serie } \\
\text { de protocolos por } \\
\text { medio de los cuales } \\
\text { pueden participar } \\
\text { en el registro de las } \\
\text { operaciones o ve- } \\
\text { rificar los cambios } \\
\text { introducidos en } \\
\text { el sistema (Preuk- } \\
\text { schat, 2017). } \\
\text { Se tiene una traza- } \\
\text { bilidad de quiénes } \\
\text { participan en el } \\
\text { sistema. }\end{array}$ & \\
\hline
\end{tabular}

BLOCKCHAIN

Y MERCADOS

FINANCIEROS:

ASPECTOS

GENERALES

DEL IMPACTO

REGULATORIO DE

LA APLICACIÓN DE

LA TECNOLOGÍA

BLOCKCHAIN EN

LOS MERCADOS

DE CRÉDITO DE

AMÉRICA LATINA

\section{BLOCKCHAIN}

AND FINANCIAL

MARKETS: GENERAL

FEATURES OF

THE REGULATORY

IMPACT OF THE

APPLICATION

OF BLOCKCHAIN

TECHNOLOGY INTHE

CREDIT MARKETS OF

LATIN AMERICA 


\section{II.5. Consenso en la cadena de bloques}

El consenso en la cadena de bloques es el elemento esencial que define el sistema blockchain. Puesto que los participantes del sistema no cuentan con una confianza plena al no conocerse, para tener certeza sobre el cumplimiento de las obligaciones, información sobre cada uno de los miembros y certeza sobre posibles fraudes, se requiere que las partes cuenten con un consenso sobre la existencia, la evolución y el estado de una serie de información compartida (Preukschat, 2017).

Es importante recalcar en este punto que el consenso depende del esquema de modelo de bloques y de si se está ante un sistema público o privado. Así, en el sistema privado, el consenso depende de la estructura preliminar que le hubiesen dado las partes participantes de dicho esquema. En otras palabras, existe una relación contractual entre los participantes, por medio de la cual se establecen las reglas de juego que regirán la relación y el intercambio de información dentro del modelo blockchain. En el sistema público -al ser un sistema descentralizado en donde no existe una autoridad central-, el consenso se convierte en un tema complejo, ya que este depende de la «confianza» de las partes participantes en el sistema blockchain y se basa en la premisa de que todas las partes son «honestas en su actuar» (Libro Blockchain, 2017).

Así, en el caso de materializarse un cambio en el sistema público, se requiere una «aceptación», la cual se materializa a través de sistemas algorítmicos. La siguiente tabla ofrece una caracterización resumida de dichos sistemas: 


\begin{tabular}{|c|c|}
\hline Algoritmo & Característica \\
\hline $\begin{array}{l}\text { Proof of } \\
\text { work }\end{array}$ & $\begin{array}{l}\text { Algoritmo hashcash. } \\
\text { Red descentralizada de tipo peer-to-peer. } \\
\text { Claves digitales asimétricas, públicas y privadas (Libro Blockchain, } \\
\text { 2017). } \\
\text { Network: es una red de nodos conectados directamente entre sí } \\
\text { (Laurence, 2017). } \\
\text { Nodos: en ellos se encuentra la información de todas las opera- } \\
\text { ciones que se realizan a través del blockchain (Laurence, 2017). } \\
\text { Cada nodo debe poseer el mismo software para que se puedan } \\
\text { comunicar entre sí (Preukschat, 2017). } \\
\text { Protocolo estándar: es el software informático por medio del } \\
\text { cual una red de nodos se puede comunicar entre sí (Preukschat, } \\
\text { 2017). } \\
\text { Sistema criptográfico: es el procedimiento a través del cual -por } \\
\text { medio de un algoritmo con clave- se transforma un mensaje sin } \\
\text { atender a su estructura lingüística o significado, lo cual implica } \\
\text { que sea incomprensible a las partes que no tengan acceso a la } \\
\text { clave secreta del algoritmo empleado (Preukschat, 2017). } \\
\text { Elevado consumo de energía de los equipos para resolver el al- } \\
\text { goritmo. } \\
\text { Minero crea una cantidad de bloques de acuerdo con la potencia } \\
\text { que tenga en el proceso, lo que puede llevar, en ciertos casos, a } \\
\text { que se concentre la función en pocas manos. }\end{array}$ \\
\hline $\begin{array}{l}\text { Proof of } \\
\text { stake }\end{array}$ & $\begin{array}{l}\text { Los bloques se crean en proporción relativa a la cantidad de token } \\
\text { que posea cada participante (Libro Blockchain, 2017): } \\
\text { Selección aleatoria. } \\
\text { Selección por antigüedad. } \\
\text { No se generan nuevos token y la recompensa se genera a través } \\
\text { de comisiones. } \\
\text { Menores costos para el minado. }\end{array}$ \\
\hline $\begin{array}{l}\text { Proof of } \\
\text { importance }\end{array}$ & $\begin{array}{l}\text { Este sistema es propio de NEM. } \\
\text { La minería no depende de la capacidad de la parte, sino de su } \\
\text { participación dentro del sistema a través de una puntuación que } \\
\text { le otorga el citado sistema (NEM, 2018). }\end{array}$ \\
\hline
\end{tabular}

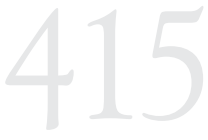

BLOCKCHAIN

Y MERCADOS

FINANCIEROS:

ASPECTOS

GENERALES

DEL IMPACTO

REGULATORIO DE

LA APLICACIÓN DE

LA TECNOLOGÍA

BLOCKCHAIN EN

LOS MERCADOS

DE CRÉDITO DE

AMÉRICA LATINA

\section{BLOCKCHAIN}

AND FINANCIAL

MARKETS: GENERAL

FEATURES OF

THE REGULATORY

IMPACT OF THE

APPLICATION

OF BLOCKCHAIN

TECHNOLOGY INTHE

CREDIT MARKETS OF

LATIN AMERICA

\section{I.6. Contratos inteligentes o smart contracts}

La idea de los contratos inteligentes o «smart contracts» surgió en la década de los 1990 con Nick Szabo, quien los describe como «un protocolo de transacción computarizado que ejecuta los términos de un contrato» (1994). Estos tienen como objetivo «satisfacer condiciones contractuales comunes (como condiciones de pago, gravámenes, confidencialidad e incluso cumplimiento), minimizar las excepciones 
maliciosas y accidentales, y minimizar la necesidad de intermediarios de confianza» (1994). Así, por «contratos inteligentes» se entiende cualquier tipo de contrato cuya característica principal es que se puede ejecutar de forma automática sin que sea necesaria la intervención de un tercero (Stokes \& Freire Ramos, 2017, p. 124).

En este sentido, los smart contracts son «scripts» almacenados dentro de la cadena de bloques. Estos scripts permiten la automatización de procesos de múltiples pasos. Así, al activar el contrato inteligente, cada nodo de la red cumple una función en la ejecución del contrato (Christidis \& Devetsikiotis, 2016, p. 2296).

En palabras del BBVA

los contratos inteligentes son scripts modulares, repetibles y autónomos que normalmente se ejecutan en un blockchain y representan promesas unilaterales de proporcionar una tarea informática determinada. Estos scripts se almacenan en el blockchain, en una dirección específica que se determina cuando se implementan los contratos en el blockchain. Cuando se produce un evento contemplado en el contrato, se envía una transacción a esa dirección y la máquina virtual distribuida ejecuta los códigos de operación del script (o cláusulas) utilizando los datos enviados con la transacción (2015).

Es importante recalcar las ventajas que poseen los contratos inteligentes frente a los contratos tradicionales, ya que, por la naturaleza de los primeros, ellos «operan de manera sencilla, rápida, inmodificable, con ejecución asegurada al no permitir el arrepentimiento y con la ventaja de que la operativa puede ser totalmente automatizada» (Echebarría Sáenz, 2017, p. 71). Mientras tanto, en los contratos tradicionales existe una serie de problemáticas tales como las modificaciones unilaterales de una de las partes (con o sin justa causa), el sometimiento de posibles interpretaciones de la voluntad de los contratantes, entre otras.

\section{APLICACIÓN DEL BLOCKCHAIN EN LOS MERCADOS FINANCIEROS}

La tecnología blockchain cuenta con un sinnúmero de aplicaciones en los mercados financieros en temas tales como inversiones, pólizas de seguros, cuentas en bancos, historiales de crédito, entre otros (Deloitte, 2017a, p. 95). A continuación, se procederá a desarrollar las principales aplicaciones de la cadena de bloques en los diferentes mercados financieros, para luego entrar a analizar con más detalle las aplicaciones de dicha tecnología en los mercados de crédito. 


\section{III.1. Mercado de valores}

Las aplicaciones de blockchain tienen un gran potencial para el mercado de valores. Los expertos en la materia sostienen que esta tecnología tiene el potencial de revolucionar las operaciones de la industria de valores, prediciendo que la adopción de la cadena de bloques se podría ver en cuestión de meses, con una adopción a gran escala en toda la industria que posiblemente ocurra después de varios años (Financial Industry Regulatory Authority, 2017, p. 1). Dentro de las aplicaciones de blockchain en el mercado de valores están las siguientes:

- Aplicación para la banca de inversión, en trading y liquidación de préstamos sindicados, los clientes corporativos podrían beneficiarse de ciclos de liquidación más cortos (Zemlianskaia, 2017, p. 38).

- Procesos de emisión de valores (Crosby, Nachiappan, Pattanayak, Verma, \& Kalyanaraman, 2015), con tecnología de bloques en la emisión de renta fija se puede programar el pago de capital y cupones (Financial Industry Regulatory Authority, 2017), al igual que en la renta variable se puede establecer registros frente al cambio de propiedad y pago de rendimientos.

- Aplicación en la etapa de cumplimiento en la negociabilidad de valores, por cuanto la tecnología blockchain podría facilitar la función de auditoría para una trazabilidad más sencilla de estas negociaciones (Committee on Payments and Market Infrastructures, 2017).

- Negociaciones postacuerdo: préstamos corporativos, credit default swaps (Financial Industry Regulatory Authority, 2017), operaciones repo ${ }^{3}$, derivados, acciones, etcétera, siendo que la tecnología blockchain podría reducir los tiempos de ejecución de estas negociaciones, por cuanto algunas de estas tardan hasta tres semanas en ejecutarse, como ocurre con los préstamos corporativos (Morgan Stanley, 2016, p. 5), siendo que la reducción en los tiempos de ejecución también puede aumentar la liquidez de las instituciones financieras.

\section{III.2. Mercado de seguros}

Las potenciales aplicaciones de la cadena de bloques en el mercado de seguros son diversas. Algunos autores manifiestan que la tecnología blockchain puede ser algún día tan común en la industria de seguros como las bases de datos de Lenguaje de Consulta Estructurada — SQL,

BLOCKCHAIN

Y MERCADOS

FINANCIEROS:

ASPECTOS

GENERALES

DEL IMPACTO

REGULATORIO DE

LA APLICACIÓN DE

LA TECNOLOGÍA

BLOCKCHAIN EN

LOS MERCADOS

DE CRÉDITO DE

AMÉRICA LATINA

BLOCKCHAIN

AND FINANCIAL

MARKETS: GENERAL

FEATURES OF

THE REGULATORY

IMPACT OFTHE

APPLICATION

OF BLOCKCHAIN

TECHNOLOGY INTHE CREDIT MARKETS OF LATIN AMERICA 
por sus siglas en inglés- (EY, 2017, p. 1). Así, dentro de las aplicaciones de blockchain en el mercado de seguros están las siguientes:

- Capacidad de verificar la propiedad de un bien asegurado o que se espera asegurar y el historial de las transacciones que sobre dichos bienes se han realizado (Crosby, Nachiappan, Pattanayak, Verma \& Kalyanaraman, 2015, p. 14).

- Prevención en materia de fraude, toda vez que las aseguradoras pueden verificar de forma independiente a los clientes y sus datos, al igual que pueden identificar las transacciones duplicadas o que sean sospechosas de generar fraudes (EY, 2017).

- Prevención y administración de reclamos, en la medida en que blockchain puede permitir que la aseguradora y varios terceros accedan y actualicen de manera fácil e instantánea información relevante (por ejemplo, formularios de reclamo, evidencias, informes policiales e informes de revisión de terceros) (EY, 2017, p. 3).

\section{III.3. Mercado de crédito}

Son muchas las posibles aplicaciones de la tecnología blockchain en los mercados de crédito, incluyendo aquellas que ya están teniendo lugar gracias a muchas iniciativas por parte de los propios bancos. Así, se puede exponer el caso de Santander, cuya iniciativa Santander InnoVentures ${ }^{4}$ lanzó, en julio de 2014, un fondo de 100 millones de dólares para investigar e implementar posibles usos del blockchain en servicios financieros, identificando más de veinte posibles usos incluyendo transferencias internacionales, préstamos sindicados y administración colateral (Williams-Grut, 17 de junio de 2015). A continuación, pasaremos a considerar los principales usos de la tecnología blockchain en los mercados de crédito.

En primer lugar, cabe mencionar los pagos transfronterizos a bajos costos (Primm, 2016, p. 81). Tómese, por ejemplo, a Ripple ${ }^{5}$ un protocolo de código abierto para las transacciones e intercambios financieros. Este protocolo tiene dos objetivos principales: el intercambio de divisas y la protección contra ataques fraudulentos a la red (Zemlianskaia, 2017, pp. 21-22). A través de Ripple se logra hacer transacciones financieras a nivel internacional en un menor tiempo y a un menor costo que en el sistema financiero actual. Así, Ripple logra reducir el costo en comisiones y pone en peligro la funcionalidad y existencia de actores como Western Union (Zemlianskaia, 2017, p. 35), pues las transferencias de dinero a cuentas bancarias a través de dicha compañía tardan entre

4 Sobre el particular, consultar el sitio web de la citada iniciativa: http://santanderinnoventures.com/

5 Sobre el particular, consultar el sitio web: https://ripple.com/ 
uno y dos días (Western Union, s.f.). Con Ripple, «[t]odo el proceso entre múltiples bancos tarda segundos y proporciona visibilidad y transparencia de la transacción de extremo a extremo, aumentando las tasas de procesamiento y bajando costes operacionales de transacción» (Zemlianskaia, 2017, p. 36). Por las ventajas que presenta Ripple, es de esperar que su expansión en el futuro sea preferida incluso por los reguladores, pues sus transferencias casi instantáneas reducen los riesgos de liquidez y de crédito que enfrentan las entidades bancarias (Blair, 2016, p. 8).

Igualmente, en los últimos años ha habido una proliferación de múltiples aplicaciones que se encargan de las remesas internacionales para aquellas personas que no tienen acceso a estos servicios financieros o que tenían que pagar una cuota bastante elevada en comparación con las cuotas que ofrecen estas aplicaciones (Mills et al., 2016, p. 19). De este modo, podemos encontrar aplicaciones como Abra y Stellar que, al reducir sustancialmente las cuotas (fees) de las remesas internacionales, ayudan a la inclusión financiera de un segmento de la población que accede a estos servicios (Biggs, 2016, pp. 193-194).

En segundo lugar, resulta pertinente referirse a los contratos inteligentes. A través de este mecanismo, se puede tener la trazabilidad de una operación de crédito, así como del trámite de un crédito con garantía real (Zemlianskaia, 2017, p. 38). Igualmente, a través de los contratos inteligentes se podría programar la ejecución y administración de empresas, concepto también conocido como las Organizaciones Autónomas Descentralizadas - DAO, por sus siglas en inglés- (Mills et al., 2016, p. 29). Las DAO son, en esencia, contratos inteligentes a largo plazo que contienen los activos y codifican los estatutos de toda una organización (Buterin, 2014). Estas han sido desarrolladas a través de Ethereum: una plataforma que ejecuta contratos inteligentes (Ethereum, 2014). De esta manera, a través de los contratos inteligentes es posible realizar procesos complejos como garantías de préstamos y contratos de futuros, e incluso la fijación de prioridades de pago en una nota estructurada (Fernández Espinosa, 16 de noviembre de 2016).

Asimismo, en los países en desarrollo, se espera que la tecnología blockchain sea un verdadero cambio de paradigma, especialmente para las pequeñas y medianas empresas (pyme). La tecnología blockchain implicaría un cambio en los actuales procesos lentos y engorrosos con mucho papeleo y obstáculos burocráticos, esta tecnología involucrará contratos inteligentes que ejecutarán transferencias automáticas de dinero a medida que se realicen envíos de mercancías a través de fronteras internacionales y eventos desencadenantes comerciales y financieros predefinidos. Se estima que esta automatización a través de la cadena de bloques pueda reducir drásticamente los costos, ampliando el acceso

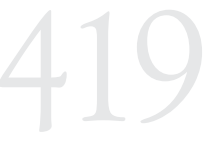

BLOCKCHAIN Y MERCADOS FINANCIEROS: ASPECTOS

GENERALES

DEL IMPACTO

REGULATORIO DE

LA APLICACIÓN DE

LA TECNOLOGÍA

BLOCKCHAIN EN

LOS MERCADOS

DE CRÉDITO DE

AMÉRICA LATINA

BLOCKCHAIN

AND FINANCIAL

MARKETS: GENERAL

FEATURES OF

THE REGULATORY

IMPACT OFTHE

APPLICATION

OF BLOCKCHAIN

TECHNOLOGY INTHE CREDIT MARKETS OF LATIN AMERICA 
financiero a las Pymes que actualmente están excluidas (Ferrarini, Maupin \& Hinojales, 2017, p. 11), ofreciéndoles más opciones (Guo \& Liang, 2016, p. 4).

En tercer lugar, han de notarse los sistemas de pago. El pago punto a punto también se puede implementar utilizando la tecnología blockchain, eliminando así intermediarios de las instituciones financieras, lo cual mejoraría enormemente la eficiencia del servicio y reduciría los costos de transacción de los bancos (Guo \& Liang, 2016, p. 6). En este sentido, la tecnología blockchain se ha extendido a las transacciones financieras y es muy probable que se utilice en sistemas financieros, incluidos los sistemas de pago, como el micropago y los sistemas de pago de gran tamaño (Yoo, 2017, p. 315). Esto tiene el potencial de propulsar un aumento en la inclusión financiera en los países en vía de desarrollo (Pilkington, 2016, p. 244), como se verá más adelante.

Según el BBVA, «en los sistemas de pago, las redes de pago a través de tarjetas de crédito y los servicios que permiten hacer transferencias están diseñados para resolver un problema de doble gasto, es decir, garantizan que no se duplique ni un solo euro y que el dinero fluya de una persona a otra. El blockchain permitiría esta garantía no solo para el dinero, sino para cualquier bien financiero que se quisiera transferir» (2016).

Una cuarta aplicación posible de la tecnología blockchain es en los procesos de supervisión financiera. A través de esta tecnología, la autoridad podría ejercer su función en muchos casos en tiempo real (Primm, 2016, p. 83). De esta forma, una cadena de bloques que contenga los datos para los reguladores podría ser más eficiente para evaluar los datos dentro de una empresa, así como para comparar los datos entre diferentes entidades reguladas (Morgan Stanley, 2016, p. 6). Además, siendo que la información almacenada en la cadena de bloques es muy difícil de corromper, podría diseñarse un «acceso de solo lectura» para los reguladores y supervisores del mercado (Mills et al., 2016, p. 20).

La tecnología blockchain también puede aplicarse en los sistemas de contabilidad financiera. El desarrollo de la tecnología blockchain y los contratos inteligentes se podría utilizar en los procesos de registro de operaciones activas y pasivas de las entidades de crédito, facilitando la contabilidad, automatizándola en una cadena de bloques. Adicionalmente, estos blockchains podrían estar disponibles como un registro público compartido entre diferentes instituciones y agencias gubernamentales, facilitando su vigilancia, cobro de impuestos, etcétera (Peters \& Panayi, 2016, p. 267). Así, se podría tener la información de los préstamos que hace una entidad financiera, con detalle de garantías y pagos realizados, y, en caso de incumplimiento de pago por el deudor, se tendría la posibilidad de revocar las claves digitales que 
permiten acceder a las garantías de forma automática e instantánea (Zemlianskaia, 2017, p. 39). Esto sería posible gracias a que se podría actualizar la información en tiempo real (Byström, 2016).

Otras aplicaciones posibles son la negociabilidad de libranzas: la empresa «PrestaGente» ${ }^{6}$ otorga créditos-libranzas, en donde la operación se lleva a cabo a través de tecnología de blockchain; y los servicios de custodia de múltiples firmas (escrow services): una de las fortalezas - y también debilidad - de la tecnología blockchain es que las transacciones son irreversibles. Para ciertos ámbitos, la irreversibilidad de las transacciones puede ser un problema, puesto que en ciertos casos puede ser necesario hacer enmiendas o correcciones sobre disputas en una negociación. Para ello, la tecnología blockchain puede aplicar un escrow service de múltiples firmas. Así, si hay una disputa entre las partes involucradas en una transacción, el escrow service puede resolver la disputa, haciendo que las transacciones no sean irreversibles (Peters \& Panayi, 2016, pp. 274-275).

Por último, es posible aplicar esta tecnología para los activos digitales. Las representaciones digitales de un activo físico, como los token y los activos digitales emitidos de forma nativa, también son componentes clave del desarrollo de la tecnología blockchain (Mills et al., 2016, p. 28).

\section{PROPUESTAS REGULATORIAS PARA LA APLICACIÓN DE BLOCKCHAIN EN LOS MERCADOS DE CRÉDITO EN AMÉRICA LATINA Y SU IMPACTO EN EL MERCADO}

\section{IV.1. Visión de un enfoque participativo por parte de los reguladores financieros y las empresas que estructuran procesos tecnológicos de blockchain}

El objetivo de la política regulatoria debería estar encaminado a propiciar el uso de la tecnología blockchain con el fin de cerrar las brechas en materia de inclusión financiera en América Latina, al igual que establecer servicios financieros más eficientes y con menores costos. No se debe perder de vista que uno de los principales problemas de la región en materia financiera son los altos índices de personas que no están dentro del sistema. Según la Federación Latinoamericana de Bancos (Felaban), para el año 2016 la relación depósitos/producto interno bruto (PIB) alcanzó un nivel promedio de $46.7 \%$ en América Latina, mientras que el cociente crédito/PIB ascendió a 44.7\% para el mismo período. Igualmente, la relación crédito/PIB ha registrado avances significativos en los últimos años, pero todavía existe un porcentaje alto de población que no puede acceder a servicios y productos financieros (Felaban, 2017).

BLOCKCHAIN Y MERCADOS FINANCIEROS: ASPECTOS GENERALES DEL IMPACTO REGULATORIO DE LA APLICACIÓN DE LA TECNOLOGÍA BLOCKCHAIN EN LOS MERCADOS DE CRÉDITO DE AMÉRICA LATINA

BLOCKCHAIN AND FINANCIAL MARKETS: GENERAL FEATURES OF THE REGULATORY IMPACT OF THE APPLICATION OF BLOCKCHAIN TECHNOLOGY INTHE CREDIT MARKETS OF LATIN AMERICA 
Bajo estos antecedentes y compartiendo la posición gremial de Felaban (2017), los avances tecnológicos en materia financiera y la penetración de la telefonía celular, el tamaño del ancho de banda, el acceso a computadores y tablets y el tráfico por usuario en megabytes pueden traer como consecuencia que se abarque un mayor número de personas en la prestación de servicios financieros. En este escenario, el blockchain sería una herramienta tecnológica de vital importancia para estos objetivos. Bajo esta premisa, los reguladores pueden tomar como política regulatoria un mecanismo participativo con las empresas Fintech, mecanismo que se ha denominado regulatory sandbox (caja de arena regulatoria).

Los sandbox se originaron en el Reino Unido, a través de su autoridad financiera, la Financial Conduct Authority (FCA). Dicha autoridad permite, en un ambiente controlado y supervisado, a las empresas probar productos, servicios, modelos comerciales y mecanismos de entrega innovadores en el mercado real, con consumidores reales (Financial Conduct Authority, 2015). Así, las cajas de arena regulatorias representan un elemento principal de los nuevos enfoques regulatorios. Los principios de las regulatory sandbox se pueden encontrar originalmente en el sector de la tecnología, donde un sandbox representa un entorno virtual para probar de forma aislada un nuevo proceso o software. Sin embargo, en el contexto de los mercados financieros, quizá sea más adecuado el paralelo con los ensayos clínicos, ya que el sector está regulado de manera similar para evitar daños al consumidor al probar las innovaciones (Arner, Barberis \& Buckley, 2017, p. 408).

Recientemente, Australia y Singapur se sumaron a la iniciativa del Reino Unido y anunciaron el establecimiento de cajas de arena reguladoras de Fintech (Guo \& Liang, 2016, p. 10). En cuanto a la FCA, esta ha establecido un marco de aplicación y salvaguardas para el funcionamiento de su sandbox. Los objetivos de mercado declarados para esta iniciativa son (1) reducir el tiempo de lanzamiento al mercado a un costo potencialmente más bajo; (2) proporcionar un mejor acceso a la financiación; y (3) fomentar productos más innovadores que lleguen al mercado (Arner, Barberis \& Buckley, 2017, p. 409).

Por otro lado, ha habido propuestas para crear estándares con respecto a la utilización de la tecnología blockchain en ámbitos financieros. Así, recientemente, Standards Australia ${ }^{7}$ ha presentado una solicitud a la Organización Internacional de Normalización (ISO) para desarrollar estándares globales para la tecnología blockchain. El consorcio blockchain R3 también está explorando la formulación de estándares industriales para aplicaciones interbancarias (Guo \& Liang, 2016, p. 10). En cuanto a Estados Unidos, la Oficina del Contralor de la Moneda (OCC, por 
sus siglas en inglés) lanzó una guía resaltando ocho principios para una futura regulación de las Fintech (Office of the Comptroller of the Currency, 2016):

1. Apoyar la innovación responsable.

BLOCKCHAIN

2. Fomentar una cultura interna receptiva a la innovación responsable.

Y MERCADOS

FINANCIEROS:

ASPECTOS

GENERALES

3. Aprovechar la experiencia y experticia de la agencia.

4. Fomentar la innovación responsable que proporcione un acceso justo a los servicios financieros y un trato justo de los consumidores.

5. Avanzar en nuevas operaciones seguras a través de una gestión de riesgos efectiva.

6. Alentar a los bancos de todos los tamaños a integrar la innovación responsable en su planificación estratégica.

7. promover el diálogo continuo a través de un alcance formal.

8. Colaborar con otros reguladores.

Así, la OCC sirve como un lugar central en donde los bancos y otras instituciones no bancarias pueden recibir una retroalimentación con respecto a las ideas innovadoras que quieran implementar sobre Fintech (Mirmazaheri, 2016, pp. 179-180). Bajo esta premisa se desarrollarán, a continuación, los principales criterios regulatorios que se deben tener en cuenta en materia de aplicación de la tecnología blockchain en los mercados financieros latinoamericanos.

\section{IV.2. Regulación en materia de riesgos}

Si bien la tecnología blockchain proporciona una gran cantidad de ventajas, su aplicación en la prestación de servicios financieros genera una serie de riesgos (EquiSoft, 2017, p. 9). Estos deben ser regulados y supervisados por las autoridades financieras, con el fin de proteger la estabilidad del sistema y a los consumidores financieros.

\section{IV.2.1. Operativos}

Partiendo de los criterios establecidos por la Financial Industry Regulatory Authority (2017), es interesante analizar algunos criterios a tener en cuenta frente a la estructura operacional del sistema. Esto permitirá identificar posibles riesgos operativos sobre la materia:

DEL IMPACTO

REGULATORIO DE

LA APLICACIÓN DE

LATECNOLOGÍA

BLOCKCHAIN EN

LOS MERCADOS

DE CRÉDITO DE

AMÉRICA LATINA

BLOCKCHAIN

AND FINANCIAL

MARKETS: GENERAL

FEATURES OF

THE REGULATORY

IMPACT OFTHE

APPLICATION

OF BLOCKCHAIN

TECHNOLOGY INTHE

CREDIT MARKETS OF

LATIN AMERICA 


\section{Tabla 5}

Criterio general

Acceso a los participantes

Validación de la transacción, teniendo en cuenta la forma de validación de la operación (ver primer apartado)

Representación del activo

Transparencia de la operación y registro de datos

\section{Puntos para mitigar los riesgos}

Criterios para establecer quién puede acceder a la red. Criterios para establecer la identidad y los antecedentes de los participantes en el sistema.

Protocolos y causales para la desvinculación de los participantes.

Protocolos para establecer quién puede tener acceso a la totalidad de la información (participante directo) y quién puede tener acceso restringido (participante indirecto).

Protocolos y posibles criterios para que el supervisor financiero pueda acceder a la información del sistema.

Consenso:

Prueba del consenso.

Se requeriría proceso algorítmico.

Fraudes por partes del consenso de varias partes del proceso.

Nodo:

¿Es necesario un solo nodo?

La copia de seguridad del nodo.

Nodos alternativos o aleatorios.

¿Cómo se corrige o rectifica una entrada errónea? ¿Cuál sería el mecanismo para tal fin?

Se emite de forma directa y se representa digitalmente. Se emite de forma tradicional y se realiza el proceso de token en el sistema. Si se extravía el activo tradicional, ¿cómo se maneja el tema?

¿Cómo se representa el dinero en la red para pagar la operación?

Como una moneda virtual.

O se utiliza moneda tradicional: icómo se realiza el pago?, ¿cómo se registra en el sistema?, ¿quién registra la operación?

¿Cómo se garantiza el pago? ¿Cuáles serían los medios o mecanismos de garantía?

¿Cuáles datos se registran?

¿Existirán datos obligatorios y opcionales?

¿Quién puede tener acceso a los datos o información que está en el sistema?

\section{IV.2.2. Informáticos o riesgos cibernéticos}

A continuación, en la siguiente tabla se exponen varios puntos relevantes en materia de seguridad (Financial Industry Regulatory Authority, 2017, p. 7). 


\begin{tabular}{|l|l|}
\hline Criterio & \multicolumn{1}{c}{ Punto para tener en cuenta } \\
\hline & $\begin{array}{l}\text { Modificación y pérdida de la clave. } \\
\text { Modificación periódica de las claves para evitar hackeos globales. } \\
\text { ¿Cuál debe ser el nivel de complejidad de la clave para evitar pro- } \\
\text { blemas informáticos? } \\
\text { ¿Cómo se revertirán las operaciones realizadas con claves compro- } \\
\text { metidas? } \\
\text { ¿Quién asume el riesgo de las operaciones realizadas con claves } \\
\text { comprometidas? }\end{array}$ \\
\hline \multirow{2}{*}{ Fraude } & $\begin{array}{l}\text { ¿Cuáles son los incentivos que se generarían para garantizar la in- } \\
\text { tegridad y seguridad en el sistema? } \\
\text { ¿Quién asume los riesgos y costos por los fraudes? } \\
\text { ¿Cuáles serían las garantías frente a las operaciones riesgosas? }\end{array}$ \\
\hline
\end{tabular}

No se debe perder de vista que los ataques se pueden dirigir a la parte más débil del sistema y no necesariamente al sistema como tal. Tal situación puede traer como consecuencia la generación de datos inexactos en el sistema, lo cual, a la postre, afectará a todo el sistema de bloques (Zetzsche, Buckley \& Arner, 2017, p. 16). Igualmente, existe un riesgo para todo el sistema de blockchain si un hacker toma el control del 51\% de los usuarios en un tiempo determinado (Deloitte, 2017b, p. 5).

Dentro de las posibles medidas para hacerle frente a problemas de ciberseguridad en la implementación de tecnología blockchain por parte de las instituciones financieras, se puede tener en cuenta las siguientes recomendaciones (Financial Industry Regulatory Authority, 2017):

- Que la institución financiera cuente con un registro de respaldo de la información de la cadena de bloques en los casos en que existan problemas de ciberseguridad que comprometan la integridad del sistema.

- Contar con protocolos internos al interior de la institución financiera con el fin de hacer pruebas para entrar a determinar los posibles riesgos en los que se ve inmersa la institución en caso de ataques cibernéticos.

- Establecer estándares de obligatorio cumplimiento para todos los participantes del sistema.

- Estándares de autenticación con múltiples factores para que los participantes puedan acceder al sistema.

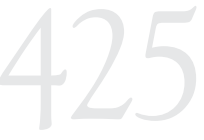

BLOCKCHAIN

Y MERCADOS

FINANCIEROS:

ASPECTOS

GENERALES

DEL IMPACTO

REGULATORIO DE

LA APLICACIÓN DE

LA TECNOLOGÍA

BLOCKCHAIN EN

LOS MERCADOS

DE CRÉDITO DE

AMÉRICA LATINA

BLOCKCHAIN

AND FINANCIAL

MARKETS: GENERAL

FEATURES OF

THE REGULATORY

IMPACT OF THE

APPLICATION

OF BLOCKCHAIN

TECHNOLOGY INTHE

CREDIT MARKETS OF

LATIN AMERICA 
- Controles de las operaciones que se realizan a través del sistema a través de umbrales de valor, número de operaciones en un tiempo determinado, entre otros.

\section{IV.2.3. Legales}

Este es uno de los puntos que mayor preocupación genera entre los reguladores financieros, ya que existe una serie de interrogantes (Zetzsche, Buckley \& Arner, 2017). Estas son algunas de las principales preocupaciones:

- iHa de darse una ley aplicable a las tecnologías de blockchain, bajo la premisa de existir una ley específica para la materia, o se deben aplicar las reglas generales en materia de contratos, bienes y derecho empresarial? (Zetzsche, Buckley \& Arner, 2017).

- Los problemas en materia de reglas de competencia y prácticas anticompetitivas por parte de los mineros en sistemas como el proof of work - situación que se corrige en cierta medida en el sistema proof of stake y en el proof of importance-.

- El tipo de participante y los requisitos para su participación, el tipo de mercado a aplicar (valores, crédito y de seguros), y el alcance local o transfronterizo (Deloitte, 2017b, p. 5).

- No se puede aplicar unas mismas reglas regulatorias al blockchain, ya que existen sistemas privados, híbridos y públicos y, dentro de este último, diferentes formas de consenso que requieren respuestas regulatorias disímiles.

- ¿Cuáles serían las reglas para determinar quién responde y bajo qué condiciones en caso de un colapso o falla en el sistema? (Zetzsche, Buckley \& Arner, 2017, p. 23).

- Que los token sean considerados como valores, en los términos de la regulación del mercado de valores (Cloots, 2018, p. 2).

Con el fin de hacerle frente a estas y otras situaciones que se generen en materia de riesgos legales, los reguladores deben trabajar junto con las empresas tecnológicas para entender el alcance de la tecnología y los retos en materia legal que dicha situación acarrea (Lewis, McPartland \& Ranjan, 2017, p. 12).

\section{IV.2.4. Liquidez}

Con el sistema, las operaciones sobre activos financieros se pueden materializar de forma muy rápida y hasta en tiempo real. Esto trae como consecuencia que se requiera mucha mayor liquidez por parte de las instituciones financieras al materializarse una reducción de las exposiciones crediticias (Committee on Payments and Market Infrastructures, 2017, p. 13). 


\section{IV.2.5. Sistémico}

Siguiendo a Cloots, la posibilidad de generarse actualmente una crisis sistémica por causa de esquemas financieros que utilicen tecnología blockchain es baja. Sin embargo, situaciones como la negociabilidad de futuros sobre Bitcoin y el crecimiento exponencial del uso de la tecnología blockchain en mercados financieros pueden generar, en el mediano plazo, cierto tipo de situaciones sistémicas que deben ser revisadas por los reguladores financieros $(2018$, p. 8).

\section{IV.3. Protección de datos}

En este punto hay que tener en cuenta la protección de los datos y el uso indebido de la información y el abuso de mercado (Zetzsche, Buckley \& Arner, 2017, p. 14). En el caso de protección de datos, las instituciones financieras que hagan uso de la tecnología blockchain deben establecer criterios para proteger la información sensible de sus clientes frente a terceros (Financial Industry Regulatory Authority, 2017, p. 16). Siguiendo a la European Securities and Markets Authority (Esma), la tecnología de bloques puede generar problemas de competencia desleal, en el caso que los participantes con mayor poder generen monopolios o restrinjan el acceso a nuevos integrantes (Esma, 2016, p. 18). Igualmente, participantes del sistema pueden hacer uso de información para manipular mercados o cometer abusos de mercado, estando frente a datos que los colocarían en una posición de ventaja frente a sus competidores (Lewis, McPartland \& Ranjan, 2017, p. 13).

\section{IV.4. Impacto de la aplicación de tecnología de blockchain en los mercados de crédito latinoamericanos}

\section{IV.4.1. Mayores índices de inclusión financiera}

Desde hace años, los países en vía de desarrollo, entre ellos los latinoamericanos, han estado rezagados en el nivel de inclusión financiera (también conocida como bancarización) de sus ciudadanos, en comparación los niveles de inclusión alcanzados en los países desarrollados. Así, la escasa bancarización de la población en los países en vía de desarrollo es un limitante para el crecimiento económico de estos países, porque implica que los individuos más necesitados no tengan acceso a los servicios financieros de la banca tradicional y, por ende, no accedan a préstamos o depósitos de dinero a través de los bancos formales ${ }^{8}$.

8 En este sentido, Tafur Saiden afirma que «[e]l concepto de bancarización tiende a relacionarse con el acceso de los agentes económicos al crédito. Sin embargo, se trata de un concepto más amplio que hace referencia al acceso a todos los servicios financieros disponibles. La bancarización es a su vez una herramienta clave para los países en vía de desarrollo, en tanto juega un papel significativo frente a la reducción de la pobreza, razón que la ha convertido en un tema de gran importancia para las economías de Latinoamérica» (2009, p. 13).

BLOCKCHAIN

Y MERCADOS

FINANCIEROS:

ASPECTOS

GENERALES

DEL IMPACTO

REGULATORIO DE

LA APLICACIÓN DE

LA TECNOLOGÍA

BLOCKCHAIN EN

LOS MERCADOS

DE CRÉDITO DE

AMÉRICA LATINA

BLOCKCHAIN

AND FINANCIAL

MARKETS: GENERAL

FEATURES OF

THE REGULATORY

IMPACT OF THE

APPLICATION

OF BLOCKCHAIN

TECHNOLOGY INTHE CREDIT MARKETS OF LATIN AMERICA 
En este sentido, varios estudios han correlacionado el nivel de inclusión financiera y el crecimiento económico de los países (véanse Burgess \& Pande, 2005; Zhan \& Sherraden, 2011; Bruhn \& Love, 2013). Así, autores como Tafur Saiden explican que «[d]ado que la bancarización está directamente relacionada con el crecimiento económico y ofrece la posibilidad a las personas de bajos ingresos de mejorar sus condiciones de vida y a las pequeñas empresas de apalancarse para desarrollar sus negocios, es claro que se constituye en una herramienta fundamental en la lucha contra la pobreza» $(2009$, p. 32).

El Banco Mundial, en su informe The Global Findex Database 2017, expone que el 31\% de los adultos están desbancarizados. Las principales barreras por las que estos adultos no han tenido acceso a los servicios financieros son la falta de dinero, los altos costos de los servicios financieros, la falta de documentación necesaria para acceder a los servicios, la falta de confianza en las instituciones financieras, etcétera. Dicho informe resalta que, a nivel global, el 26\% de los adultos desbancarizados dicen que los altos costos de los servicios financieros no les permiten acceder a dichos servicios. Pero esta razón es más preocupante para el caso latinoamericano, en donde se duplica la anterior cifra. Específicamente para los casos de Brasil, Colombia y Perú, el 60\% de los adultos desbancarizados explican que son los altos costos los que les impiden acceder a los servicios financieros (Demirgüç-Kunt, Klapper, Singer, Ansar \& Hess, 2018, pp. 39-40).

Debido a lo anterior, en América Latina ciertos gobiernos han empezado a debatir posibles soluciones para lograr una mayor inclusión financiera de sus ciudadanos. Así, México, Brasil y Colombia son los pioneros en elaborar, desde hace algunos años, informes anuales de inclusión financiera y en incluir en sus agendas como tema prioritario la bancarización (Roa, 2013, p. 123). Si bien es cierto que en la región todavía existen brechas en materia de acceso a tecnologías para ciertos sectores poblacionales, dicha situación ha venido disminuyendo en los últimos años (Felaban, 2017, p. 17; Comisión Económica para América Latina y el Caribe (Cepal), 2016). Es así como muchos Estados de la región han implementado, dentro de sus políticas públicas, la política de aumentar la cobertura en materia tecnológica a diferentes nichos poblacionales, en especial a comunidades aisladas o con escasos recursos (MinTic, 2018).

Ahora bien, la tecnologíablockchain puede reducir considerablemente los costos de los servicios financieros, facilitando así la inclusión financiera de la población desbancarizada en América Latina. Específicamente, la tecnología blockchain puede reducir los costos de transacción en su faceta de costos de información. En la medida en que una cadena de bloques es gran registro contable distribuido, descentralizado y público, 
la información sobre las transacciones o posibles transacciones está a la disponibilidad de cualquier agente.

Creemos que blockchain transformará la forma en que se organizan y administran las empresas. Este permite a las compañías eliminar los costos de transacción y usar recursos en el exterior tan fácilmente como los recursos en el interior. La integración vertical puede seguir teniendo sentido en algunas situaciones (para la fabricación de productos farmacéuticos controlados, por ejemplo, o donde las empresas tienen fortalezas líderes en la industria a lo largo de la cadena de suministro). Pero en la mayoría de los casos, creemos que las redes basadas en blockchain serán más adecuadas para crear productos y servicios y para ofrecer valor a las partes interesadas (Tapscott \& Tapscott, 2017, p. 11).

Específicamente en el tema de bancarización, autores como Pilkington resaltan la gran importancia de la tecnología blockchain en la inclusión en los servicios financieros de una gran parte de la población que no está bancarizada. Así, Pilkington expone que «[u]na de las aplicaciones más emocionantes e imprevistas [de blockchain] se refiere a la inclusión social en el mundo en desarrollo, más notablemente de la población no bancarizada, es decir, todos los adultos en el mundo que no tienen una cuenta en un banco, institución financiera o un proveedor de servicios de dinero móvil» (2016, p. 244).

Igualmente, Larios-Hernández considera que, dado que «los servicios financieros tales como el crédito, los seguros o las instalaciones de ahorro son de naturaleza distinta a los servicios de pago (Donovan, 2012), las oportunidades de blockchain se derivan de la capacidad de la tecnología para operar tokens que otorgan acceso a una cartera alternativa de servicios financieros que el sistema financiero actual es incapaz de satisfacer» $(2017$, p. 866).

\section{IV.4.2. Aumento de la eficiencia}

La tecnología blockchain tiene el potencial de reducir los costos de transacción y las asimetrías de información que se presentan en los mercados de crédito. Así, esta tecnología genera eficiencias que pueden implicar la entrada al mercado y acceso a los recursos financieros por parte de un alto porcentaje de la población latinoamericana que actualmente carece de acceso. En este sentido, tal como explica Ronald H. Coase en su Conferencia del Nobel de Economía de 1991,

[s] los costos de hacer un intercambio son mayores que los beneficios que produce, esta transacción no ocurre y la mayor producción que surgiría, debida a la especialización, no sería reconocida. De esta forma, los costos de la transacción no solo afectan arreglos contractuales sino también cuáles bienes y servicios se producen. Al no incluir los costos de transacción en la teoría se dejan muchos aspectos del funcionamiento

BLOCKCHAIN Y MERCADOS FINANCIEROS: ASPECTOS

GENERALES

DEL IMPACTO

REGULATORIO DE

LA APLICACIÓN DE

LA TECNOLOGÍA

BLOCKCHAIN EN

LOS MERCADOS

DE CRÉDITO DE

AMÉRICA LATINA

BLOCKCHAIN

AND FINANCIAL

MARKETS: GENERAL

FEATURES OF

THE REGULATORY

IMPACT OF THE

APPLICATION

OF BLOCKCHAIN

TECHNOLOGY INTHE CREDIT MARKETS OF LATIN AMERICA 
del sistema económico sin explicación, incluyendo la aparición de la firma, pero mucho más aún (2012, pp. 23-24).

Así, por los altos costos de transacción que experimentan tanto los bancos como gran parte de la población latinoamericana (especialmente la de bajos recursos), no se producen los negocios por los cuales estas personas acceden a los servicios financieros de los bancos. Esto lo detalla Larios-Hernández: «algunos estudios explican el bajo nivel de inclusión financiera con variables monetarias relacionadas con altos costos de transacción y pobreza» $(2017$, p. 870).

Pero más aún, la tecnología blockchain tiene el potencial de competir contra los bancos como organización. Para explicar cómo esto es posible, tenemos que analizar a los bancos como firmas que, en el sentido de Coase, surgen «principalmente por la existencia de costes producidos por utilizar el mercado» (1937). Los bancos son intermediarios que se encargan de unir la oferta y la demanda de capital, en situaciones en las que sin el intermediario muchas de esas transacciones no se llevarían a cabo por los altos costos de transacción. Así, la tecnología blockchain es una tecnología disruptiva pues, al unir directamente a los vendedores de capital (los que de otra forma realizarían depósitos en un banco) con los compradores de capital (los que de otra manera buscarían préstamos de los bancos), esta tecnología peer-to-peer amenaza directamente a los bancos como intermediarios (MacDonald, Allen \& Potts, 2016, p. 281).

Así, los autores en la materia han hablado del fin de los bancos, debido a que esta tecnología hace posible reducir e incluso eliminar a los intermediarios en las relaciones financieras: «El $\mathrm{DLT}^{9}$ surge a raíz de una tendencia creciente de desintermediación, de la cual un síntoma es la creciente participación del mercado de la banca paralela dentro de las finanzas globales, una tendencia que incluso ha llevado a algunos a llamarla el "fin de la banca"»(Collomb \& Sok, 2016, p. 96). Estos mismos autores han sustentado que la tecnología blockchain puede mejorar la eficiencia de los mercados financieros sustancialmente, provocando un cambio de paradigma en los modelos tradicionales de mercados financieros y los mismos mercados con tecnología blockchain. Así, Collomb y Sok afirman lo siguiente: «volviendo al trabajo de Porter sobre las fuerzas competitivas, DLT puede considerarse indistintamente como una amenaza en las situaciones establecidas, pero también como una oportunidad para una mayor eficiencia» (2016, p. 97).

Por lo demás, cabe decir que la tecnología blockchain puede reorganizar la forma como funcionan los bancos. Esto se debe a que dicha tecnología fundamentalmente reordenará la gobernanza de la

9 Distributed-Ledger Technology, término que es un sinónimo de blockchain, pues la tecnología blockchain es un registro contable distribuido. 
producción de servicios bancarios, compitiendo con los bancos como organizaciones, permitiendo que las transacciones bancarias se desvíen de las organizaciones jerárquicas centralizadas y vuelvan a los mercados descentralizados (MacDonald, Allen \& Potts, 2016).

Asimismo, la tecnología blockchain tiene el potencial de reducir las asimetrías de información existentes entre los bancos, los usuarios y el regulador. La información asimétrica «[s]e basa en la idea de que uno de los dos actores involucrados en el proceso desconoce información relevante que el otro actor sí posee, de tal forma que esta falta de información implica obstáculos para que el mercado del bien o servicio en cuestión trabaje eficientemente, y en consecuencia se generan costos adicionales, insuficiencias o racionamientos que de no existir la mencionada asimetría de la información no ocurrirían» (ChabléSangeado, 2006).

Debido a lo anterior, los mercados no llegan a ser eficientes en la asignación delos recursos. Asimismo, debidoalainformaciónasimétrica, pueden darse incentivos de abusos sobre dichas asimetrías. Así, Stiglitz reconoce que, en general, «[1]os mercados están plagados de problemas de asimetrías de información, y existen incentivos para que los participantes exploten y aumenten esas asimetrías. Por diversas razones, hacen falta mercados claves (como los de seguros contra algunos riesgos importantes que afrontan los individuos y las firmas [...])»(2010, p. 14). Cabe anotar que las asimetrías de información no solo se presentan entre los bancos y sus usuarios, sino también entre estos y las entidades que los regulan. Así, Stiglitz advierte que, para corregir estas asimetrías, el regulador debe tener en cuenta que existe una asimetría de información respecto del mismo, por cuanto este no posee toda la información necesaria para entrar a intervenir el mercado de manera eficiente para corregir las fallas de mercado. Así, el autor afirma que «[e]l diseño de estructuras y sistemas de regulación debe tomar en cuenta: las asimetrías de información, porque el regulador suele tener desventajas de información con respecto a los que regula» (2010, p. 16).

Por otra parte, Stiglitz menciona que la información asimétrica no solo se origina por una falta de información de alguna de las partes dentro del mercado, sino también en la complejidad de dicha información. Esto es el caso especialmente en el mercado financiero donde, si bien las partes pueden tener toda la información relevante para el adecuado funcionamiento del mercado, dicha información es muy compleja para una de las partes (especialmente para los consumidores): «las regulaciones estándar de la transparencia por sí mismas pueden no ser suficientes. El problema de muchos derivados fue que eran tan complejos que así se hubiera revelado toda la información relevante, la mayoría de

BLOCKCHAIN Y MERCADOS FINANCIEROS: ASPECTOS

GENERALES

DEL IMPACTO

REgulatorio DE

LA APLICACIÓN DE

la TECNOLOGÍA

BLOCKCHAIN EN

LOS MERCADOS

DE CRÉDITO DE

AMÉRICA LATINA

BLOCKCHAIN

AND FINANCIAL

MARKETS: GENERAL

FEATURES OF

THE REGULATORY

IMPACT OF THE

APPLICATION

OF BLOCKCHAIN

TECHNOLOGY INTHE CREDIT MARKETS OF LATIN AMERICA 
los participantes en el mercado no habría podido determinar su valor real» (2010, pp. 18-19).

En consecuencia, existen asimetrías de información en la población, incluyendo la no bancarizada, y en el regulador dentro de los mercados financieros. Además, la población no solo es vulnerable por dichas asimetrías, sino que incluso cuando tiene la información disponible, esta no resulta fácil de entender por su complejidad. En este contexto, se debe resaltar que la tecnología blockchain tiene el potencial de reducir significativamente tanto los costos de transacción como las asimetrías de información, como ya se ha venido tratando en el desarrollo del presente trabajo.

Así, la tecnología blockchain sirve, sobre todo, para tener una trazabilidad fidedigna de todas las transacciones hechas en un determinado mercado. Esto podría ayudar a mitigar enormemente las asimetrías de información, debido a que cualquier individuo puede consultar la trazabilidad de determinada transacción de su interés, sabiendo todos los detalles de una manera sencilla y sin que la búsqueda de la información suponga altos costos de transacción. En este sentido, Collomb y Sok han expresado lo siguiente: «La capacidad de DLT de rastrear la propiedad de forma distribuida e inmutable ha sido atractiva tanto para emisores como para inversores, como parece confirmar la iniciativa Nasdaq Linq» (2016, p. 102). Cabe resaltar que a través del mundo ya se está empezando a aplicar estas tecnologías, haciendo de los mercados financieros los lugares en donde se experimenta con blockchain:

En los últimos dos años, se han lanzado muchas asociaciones entre instituciones financieras y nuevas empresas de DLT, y estos anuncios han sacudido la percepción generalmente conservadora de la banca tradicional. Queda por ver si estas empresas de DLT terminan reestructurando el sector financiero de la misma forma que los unicornios están transformando otros sectores de la economía. Pero es una señal muy fuerte de que estas iniciativas usualmente han inscrito a nombres familiares en las finanzas, y no solo del sector privado (pp. 107-108).

\section{CONCLUSIONES}

Blockchain representa una verdadera revolución en la forma de transmitir y procesar información, ya que es un mecanismo por medio del cual se reducen costos y se materializan procesos eficientes con respuesta en tiempo real. En este sentido, lo que empezó como un mecanismo que soporta los esquemas de criptomonedas cuenta con un sinfín de aplicaciones en procesos del sector público y del sector privado, sin que los mercados financieros sean la excepción. De esta manera, los mercados de valores, de seguros y de crédito pueden obtener múltiples 
ventajas tanto para sus procesos internos como para la prestación de sus servicios, trayendo como consecuencia la reducción de costos y mejores condiciones para los consumidores en la prestación de servicios.

No obstante, surge una serie de temáticas que deben ser analizadas por las autoridades financieras con respecto a la implementación del blockchain en los mercados financieros. De este modo, temas como la administración de riesgos de liquidez, riesgos cibernéticos, legales, operativos y sistémicos en la implementación de dicha tecnología deben estar dentro de las prioridades de los reguladores locales y de las entidades de la arquitectura financiera internacional. También debe ser prioritaria la protección de datos personales y la prevención de conductas anticompetitivas.

Sin embargo, pese a los riesgos, esta tecnología también representa un sinnúmero de ventajas tanto para buscar mecanismos más eficientes de inclusión financiera y para reducir los costos en la prestación de servicios financieros. Así, considerando el elevado porcentaje de personas que no están dentro del sistema financiero en América Latina, el blockchain podría ser una herramienta útil para lograr la bancarización de la población en dicha región. De esta forma, como explican Collomb y Sok, «[1] tecnología de la información ha jugado un papel clave en el desarrollo de servicios financieros modernos. [...] hoy las instituciones financieras dependen en gran medida de grandes sistemas de información y bases de datos para llevar a cabo sus negocios, cualquiera que sea el campo - titulización, derivados, o simplemente banca comercial, para nombrar unos pocos» (2016, p. 93).

Se propone que, para la materialización de esta propuesta, los Estados de la región implementen, en primer lugar, un marco regulatorio por medio del cual se autorice la prestación de servicios financieros a través de blockchain a instituciones financieras y el otorgamiento de créditos con recursos propios a particulares, se estructure los criterios tecnológicos, la administración de riesgos, los estándares de protección a los consumidores y las formas de supervisión. En segundo lugar, cada Estado de América Latina debe fortalecer las políticas públicas ya existentes en materia de inclusión tecnológica para la población, ya que, si bien existen grandes avances en materia de cobertura de internet, telefonía móvil, ancho de banda y entrega de tablets y laptops, se siguen presentando brechas digitales para un importante porcentaje de la población.

Finalmente, se conmina a los lectores de este documento a profundizar en su contenido para que, en un futuro cercano, se puedan elaborar estados de la cuestión y propuestas regulatorias que dinamicen la aplicación del blockchain en los mercados financieros de América Latina.

BLOCKCHAIN Y MERCADOS FINANCIEROS: ASPECTOS

GENERALES

DEL IMPACTO

REGULATORIO DE

LA APLICACIÓN DE

LA TECNOLOGÍA

BLOCKCHAIN EN

LOS MERCADOS

DE CRÉDITO DE

AMÉRICA LATINA

BLOCKCHAIN

AND FINANCIAL

MARKETS: GENERAL

FEATURES OF

THE REGULATORY

IMPACT OFTHE

APPLICATION

OF BLOCKCHAIN

TECHNOLOGY INTHE CREDIT MARKETS OF LATIN AMERICA 


\section{REFERENCIAS}

Arner, D.W., Barberis, J. \& Buckley, R.P. (2017). FinTech, RegTech, and the Reconceptualization of Financial Regulation. Northwestern Journal of International Law $\mathcal{E}$ Business, 37(3), 371-413. Recuperado de https://scholarlycommons.law. northwestern.edu/njilb/vol37/iss3/2/

BBVA. (2015). Smart contracts: ilo último en automatización de la confianza? Madrid: BBVA Research.

BBVA. (2016). «Blockchain» quiere ser un aliado de los servicios financieros. BBVA. Recuperado de https://www.bbva.com/es/blockchain-reescribe-las-reglaslos-servicios-financieros/

Biggs, D.C. (2016). How Non-Banks Are Boosting Financial Inclusion and Remittance. En P. Tasca, T. Aste, L. Pelizzon y N. Perony (eds.), Banking Beyond Banks and Money: A Guide to Banking Services in the Twenty-First Century (pp. 181196). Suiza: Springer International Publishing. https://doi.org/10.1007/978-3319-42448-4_10

Blair, D. (2016). Ripple vs SWIFT: Payment (r)evolution. Recuperado de https:// www.atc.asia/articles/170105/aca161124ripple.pdf

Bruhn, M. \& Love, I. (2013). The Economic Impact of Expanding Access to Finance in Mexico. En R. Cull, A. Demirgüç-Kunt y J. Morduch (eds.), Banking the World: Empirical Foundations of Financial Inclusion (pp. 137-156). MIT Press. Recuperado de http://www.jstor.org/stable/j.ctt5vjqzp.8

Burgess, R. \& Pande, R. (2005). Can Rural Banks Reduce Poverty? Evidence from the Indian Social Banking Experiment. American Economic Review, 95(3), 780-795. Recuperado de http://scholar.harvard.edu/files/rpande/files/ can_rural_banks_reduce_poverty_evidence_from_the_indian_social_banking_ experiment.pdf

Buterin, V. (2014). A Next-Generation Smart Contract and Decentralized Application Platform. Recuperado de https:/github.com/ethereum/wiki/wiki/ White-Paper\#decentralized-autonomous-organizations

Byström, H. (2016). Blockchains, Real-Time Accounting and the Future of Credit Risk Modeling. Working Paper 2016:4, Lund University, Suecia. Recuperado de https:/project.nek.lu.se/publications/workpap/papers/wp16_4.pdf

Chablé-Sangeado, J. (2006). Information Theories and Functioning of Credit Market. Hitos de Ciencias Económico Administrativa, 12(34), 127-138.

Christidis, K. \& Devetsikiotis, M. (2016). Blockchains and Smart Contracts for the Internet of Things. IEEE Access, 4, 2292-2303. https://doi.org/10.1109/ access.2016.2566339

Cloots, A. (2018). Cryptocurrencies, Blockchain and Risk Management: Legal, Operational and Systemic Risks. Cambridge, McKinsey Risk Prize. Recuperado de https://www.jbs.cam.ac.uk/fileadmin/user_upload/research/centres/risk/ downloads/riskprize2018-cloots.pdf 
Coase, R.H. (1937). The Nature of the Firm. Economica, 4(16), 386-405. https:// doi.org/10.1111/j.1468-0335.1937.tb00002.x

Coase, R.H. (2012). La estructura institucional de producción. Revista Universidad EAFIT, 28(85), 19-27. Recuperado de http://publicaciones.eafit.edu.co/index. php/revista-universidad-eafit/article/view/1485

Collomb, A. \& Sok, K. (2016). Blockchain/Distributed Ledger Technology (DLT): What Impact on the Financial Sector? Digiworld Economic Journal, 103, 93-111.

Comisión Económica para América Latina y el Caribe (Cepal). (2016). Aumenta fuertemente el uso y el acceso a Internet en América Latina y el Caribe. Santiago de Chile: Cepal.

Committee on Payments and Market Infrastructures. (2017). Distributed Ledger Technology in Payment, Clearing and Settlement: An Analytical Framework. Bank for International Settlements. Recuperado de https://www.bis.org/cpmi/publ/d157. pdf

Crosby, M., Nachiappan, Pattanayak, P., Verma, S. \& Kalyanaraman, V. (2015). BlockChain Technology: Beyond Bitcoin. AIR Applied Innovation Review, 2, 7-19. Recuperado de https://scet.berkeley.edu/wp-content/uploads/BlockchainPaper. pdf

Deloitte. (2017a). Blockchain: Economía de confianza. Tomando el control de la identidad digital. Recuperado de https:/www2.deloitte.com/content/dam/ Deloitte/co/Documents/audit/BibliotecaTecnica/RecursosAuditoria/Cinetica/ Cinética\%207.pdf

Deloitte. (2017b). Blockchain Risk Management: Risk Functions Need to Play An Active Role in Shaping Blockchain Strategy. Nueva York: Deloitte. Recuperado de https://www2.deloitte.com/content/dam/Deloitte/us/Documents/financialservices/us-fsi-blockchain-risk-management.pdf

Demirgüç-Kunt, A.; Klapper, L.; Singer, D.; Ansar, S. \& Hess, J. (2018). The Global Findex Database 2017: Measuring Financial Inclusion and the Fintech Revolution. Washington D.C.: World Bank. https://doi.org/10.1596/978-1-4648-1259-0

Echebarría Sáenz, M. (2017). Contratos electrónicos autoejecutables (smart contract) y pagos con tecnología blockchain. Revista de Estudios Europeos, 70, 69. 97. Recuperado de http://uvadoc.uva.es/handle/10324/28434

EquiSoft. (2017). La cadena de bloques (blockchain): Una tecnología disruptiva con el poder de revolucionar el sector financiero. EquiSoft.

European Securities and Markets Authority (Esma). (2016). The Distributed Ledger Technology Applied to Securities Markets. París: Esma.

Ethereum. (2014). Build Unstoppable Applications: Ethereum. Recuperado de https://www.ethereum.org/ 
EY. (2017). Blockchain in Insurance: Applications and Pursuing a Path to Adoption. Recuperado de https://www.ey.com/Publication/vwLUAssets/EY-blockhain-ininsurance/\$FILE/EY-blockhain-in-insurance.pdf

Federación Latinoamericana de Bancos (Felaban). (2017). III Informe de inclusión financiera Felaban 2017. Bogotá: Felaban.

Fernández Espinosa, L. (16 de noviembre de 2016). Qué son los 'smart contracts' o contratos inteligentes. BBVA. Recuperado de https://www.bbva.com/es/smartcontracts-los-contratos-basados-blockchain-no-necesitan-abogados/

Ferrarini, B., Maupin, J. \& Hinojales, M. (2017). Distributed Ledger Technologies for Developing Asia. Asia Development Bank Economics Working Paper Series, 533, 1-27. https://doi.org/10.22617/wps179184-2

Financial Conduct Authority. (2015). Regulatory sandbox. Financial Conduct Authority. Recuperado de https://www.fca.org.uk/firms/regulatory-sandbox

Financial Industry Regulatory Authority. (2017). Distributed Ledger Technology: Implications of Blockchain for the Securities Industry. Washington D.C.: FINRA.

Government Office for Science. (2016). Distributed Ledger Technology: Beyond Block Chain. UK Government Chief Scientific Adviser. Londres: Government Office for Science. Recuperado de https://assets.publishing.service.gov.uk/ government/uploads/system/uploads/attachment_data/file/492972/gs-16-1distributed-ledger-technology.pdf

Guo, Y. \& Liang, C. (2016). Blockchain Application and Outlook in The Banking Industry. Financial Innovation, 2(24), 1-12. https://doi.org/10.1186/s40854-0160034-9

Gustavo Cano, C.; Esguerra Umaña, M.; García, N.; Rueda, J. \& Velasco, A. (2014). Inclusión financiera en Colombia. Recuperado de www.banrep.gov.co/sites/ default/files/eventos/archivos/sem_357.pdf

Institute of International Finance. (2015). Banking on the Blockchain: Reengineering the Financial Architecture. París: Institute of International Finance.

Larios-Hernández, G. (2017). Blockchain Entrepreneurship Opportunity in the Practices of the Unbanked. Business Horizons, 60(6), 865-874. https://doi. org/10.1016/j.bushor.2017.07.012

Laurence, T. (2017). Blockchain for Dummies. Nueva Jersey: John Willey \& Sons.

Lewis, R.; McPartland, J. \& Ranjan, R. (2017). Blockchain and Financial Market Innovation. Economic Perspectives, 7, 2-12. Recuperado de https:/www. chicagofed.org/ /media/publications/economic-perspectives/2017/ep2017-7pdf.pdf

Libro Blockchain. (2017). Sitio web, versión actualizada al 29 de mayo de 2017. Recuperado de http://ibroblockchain.com/consenso/

MacDonald, T.J., Allen, D.W. \& Potts, J. (2016). Blockchains and the Boundaries of Self-Organized Economies: Predictions for the Future of Banking. En P. Tasca, T. Aste, L. Pelizzon y N. Perony (eds.), Banking Beyond Banks and Money. A Guide 
to Banking Services in the Twenty-First Century (pp. 279-296). Suiza: Springer International Publishing. https://www.doi.org/10.1007/978-3-319-42448-4 14

Mills, D.; Wang, K.; Malone, B.; Ravi, A.; Marquardt, J.; Chen, C.; Badev, A.; Brezinski, T.; Fahy, L.; Liao, K.; Kargenian, V.; Ellithorpe, M. Ng. W. \& Baird, M. (2016). Distributed ledger technology in payments, clearing, and settlement. Finance and Economics Discussion Series 2016-095. Washington: Board of Governors the Federal Reserve System. https://doi.org/10.17016/FEDS.2016.095

MinTic. (2018). Boletín trimestral de las TIC. Bogotá: MinTic.

Mirmazaheri, S. (2016). How FinTech Firms Provide a New Path to Regulatory Relief for Banks. Review of Banking $\mathcal{G}$ Financial Law, 36, 175-194. Recuperado de https://www.bu.edu/rbfl/files/2017/03/DA-14.pdf

Morgan Stanley. (2016). Global Insight: Blockchain in Banking: Disruptive Threat or Tool? Recuperado de https://www.febelfin.be/sites/default/files/ InDepth/310016810-morgan-stanley-blockchain-report_0.pdf

Natarajan, H., Krause, S. \& Gradstein, H. (2017). Distributed Ledger Technology (DLT) and Blockchain. Washington D.C.: World Bank. https:/doi. org/10.1596/29053

NEM. (2018). NEM Technical Reference. NEM.

Office of the Comptroller of the Currency. (2016). Supporting Responsible Innovation in the Federal Banking System: An OCC Perspective. Washington D.C.: Autor

Pacheco Jiménez, M.N. (2016). Criptodivisas: del bitcoin al MUFG. El potencial de la tecnología blockchain. Revista CESCO de Derecho de Consumo, 19, pp. 6-15. Recuperado de https://www.revista.uclm.es/index.php/cesco/article/ view/1180/952

Pastor Sempere, M.C. (2017). Criptodivisas: ¿Una disrupción jurídica en la Eurozona? Revista de Estudios Europeos, 70, 284-318. Recuperado de http://www. ree-uva.es/images/numeros/70/2017-70-284-318.pdf

Peters, G.W. \& Panayi, E. (2016). Understanding Modern Banking Ledgers through Blockchain Technologies: Future of Transaction Processing and Smart Contracts on the Internet of Money. En P. Tasca, T. Aste, L. Pelizzon y N. Perony (eds.), Banking beyond Banks and Money: A Guide to Banking Services in the TwentyFirst Century (pp. 239-278). Suiza: Springer International Publishing. https:// www.doi.org/10.1007/978-3-319-42448-4_13

Pilkington, M. (2016). Blockchain Technology: Principles and Applications. En F. Olleros \& M. Zhegu (eds.), Research Handbook on Digital Transformations (pp. 225-253). Cheltenham: Edward Elgar Publishing. https://doi. org/10.4337/9781784717766.00019

Preukschat, A. (2017). Blockchain: la revolución industrial de Internet. España: Ediciones Gestion 2000. 
Primm, H. (2016). Regulating the Blockchain Revolution: A Financial Industry Transformation. Review of Banking Eु Financial Law, 36, 75-91. Recuperado de https://www.bu.edu/rbfl/files/2017/03/DA-7.pdf

Roa, M.J. (2013). Inclusión financiera en América Latina y el Caribe: acceso, uso y calidad. Boletín del CEMLA, 59(3), 121-148.

Rochina, P. (2017). ¿Qué es blockchain?: principios y funcionamiento. Revistadigital INESEM. Recuperado de https://revistadigital.inesem.es/informatica-y-tics/ blockchain/

Stiglitz, J. (2010). Regulación y fallas. Revista de Economía Institucional, 12 (23), 13 28. Recuperado de https://revistas.uexternado.edu.co/index.php/ecoins/article/ view/2495/2133

Stokes, M. \& Freire Ramos, G. (2017). Smart Contracts. Actualidad Jurídica Uría Menéndez, 46, 124-127. Recuperado de https://www.uria.com/documentos/ publicaciones/5459/documento/foro_port02.pdf?id=7139

Swan, M. (2015). Blockchain: Blueprint for a New Economy. Sebastopol: O'Reilly Media.

Szabo, N. (1994). Smart Contracts. Recuperado de http://www.fon.hum.uva.nl/ rob/Courses/InformationInSpeech/CDROM/Literature/LOTwinterschool2006/ szabo.best.vwh.net/smart.contracts.html

Tafur Saiden, C. (2009). Bancarización: una aproximación al caso colombiano a la luz de América Latina. Estudios Gerenciales, 25(110), 13-37. https://doi. org/10.1016/s0123-5923(09)70060-9

Tapscott, D. \& Tapscott, A. (2017). How Blockchain Will Change Organizations. MIT Sloan Management Review, 58(2), 10-13. Recuperado de https://sloanreview. mit.edu/article/how-blockchain-will-change-organizations/

The Financial Action Task Force. (2015). Guidance for a Risk-Based Approach: Virtual Currencies. París: The Financial Action Task Force.

U.S. Securities and Exchange Commission (SEC). (2018). Initial Coin Offerings. Washington: SEC.

Western Union. (s.f.). Frequently Asked Questions: Western Union. Western Union. Recuperado de https://www.westernunion.com/it/en/faq-send-moneyonline.html

Williams-Grut, O. (17 de junio de 2015). Santander Is Experimenting with Bitcoin and Close to Investing in a Blockchain Startup. Business Insider UK. Recuperado de http://uk.businessinsider.com/santander-has-20-25-use-cases-forbitcoins-blockchain-technology-everyday-banking-2015-6

World Economic Forum. (2018). Blockchain Beyond the Hype: A Practical Framework for Business Leaders. Ginebra: World Economic Forum.

Yoo, S. (2017). Blockchain Based Financial Case Analysis and Its Implications. Asia Pacific Journal of Innovation and Entrepreneurship, 11 (3), 312-321. https://doi. org/10.1108/apjie-12-2017-036 
Zemlianskaia, A. (2017). Tecnología blockchain como palanca de cambio en el sector financiero y bancario (tesis de máster universitario en Estudios Avanzados en Dirección de Empresas). Universidad de Sevilla, España. Recuperado de https://idus.us.es/xmlui/bitstream/handle/11441/71904/Tecnolog\%C3\%ADa Blockchain_como_palanca_de_cambio.pdf?sequence $=1$ \& isAllowed $=\mathrm{y}$

BLOCKCHAIN

Zetzsche, D.; Buckley, R. \& Arner, D. (2017). The Distributed Liability of Distributed Ledgers: Legal Risks of Blockchain. University of New South Wales Law Research Series, 1-43. https://doi.org/10.2139/ssm.3018214

Zhan, M. \& Sherraden, M. (2011). Assets and Liabilities, Educational Expectations, and Children's College Degree Attainment. Children and Youth Services Review, 33(6), 846-854. https://doi.org/10.1016/j.childyouth.2010.12.006

Y MERCADOS

FINANCIEROS:

ASPECTOS

GENERALES

DEL IMPACTO

REGULATORIO DE

LA APLICACIÓN DE

LA TECNOLOGÍA

BLOCKCHAIN EN

LOS MERCADOS

DE CRÉDITO DE

AMÉRICA LATINA

BLOCKCHAIN

AND FINANCIAL

MARKETS: GENERAL

FEATURES OF

THE REGULATORY

IMPACT OF THE

APPLICATION

OF BLOCKCHAIN

TECHNOLOGY INTHE

CREDIT MARKETS OF

LATIN AMERICA

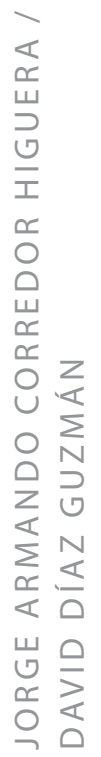

